\title{
Enquête
}

Archives de la revue Enquête

5 | 1989

Biographie et cycle de vie

\section{La définition des âges de la vie}

Une question de rapports intergénérationnels

\section{Francis Godard}

\section{(2) OpenEdition}

Journals

Édition électronique

URL : http://journals.openedition.org/enquete/84

DOI : 10.4000/enquete.84

ISSN : 1953-809X

Éditeur :

Cercom, Éditions Parenthèses

Édition imprimée

Date de publication : 2 mars 1989

Référence électronique

Francis Godard, «La définition des âges de la vie », Enquête [En ligne], 5 | 1989, mis en ligne le 27 juin 2013, consulté le 19 avril 2019. URL : http://journals.openedition.org/enquete/84 ; DOI : 10.4000/ enquete.84

Ce document a été généré automatiquement le 19 avril 2019 


\title{
La définition des âges de la vie
}

\author{
Une question de rapports intergénérationnels
}

\section{Francis Godard}

1 Ce texte s'inscrit dans un effort plus général de développement et d'approfondissement de l'approche biographique. On développe ici l'idée qu'il ne suffit pas de considérer le déroulement temporel des histoires de vie d'un point de vue purement endogène. Autrement dit que l'on ne peut s'en tenir au seul point de vue de l'économie interne ou de l'auto-engendrement des pratiques individuelles (dire que l'on ne peut s'en tenir là ne signifie d'ailleurs aucunement qu'il n'est pas nécessaire de le faire aussi). Nous développerons trois idées : a) L'organisation des vies singulières est structurée par des formes sociales d'organisation de l'existence ; apparaissent alors les concepts de cycle de vie et d'âge de la vie. b) Les modes de déroulement de l'existence dépendent des périodes historiques au cours desquelles les individus connaissent les différents moments de leur cycle de vie et apparait ici le concept de génération $\left.{ }^{1}, c\right)$ Enfin, le déroulement du cycle de vie est fortement influencé par les rapports qui se nouent entre les générations qui sont de l'ordre de la lutte pour l'occupation des places sociales, de la transmission, de la socialisation.

2 Nous serons amené ici à nous interroger sur les rapports sociaux intergénérationnels (on notera RIG), c'est-à-dire sur des rapports sociaux qui mettent en jeu des intérêts ou des attributs sociaux liés à la position sur le cycle de vie et aux processus idéologiques et culturels à partir desquels s'élaborent les représentations des attributs de chaque « âge de la vie».

3 Ceci revient en particulier à s'interroger sur les modes de régulation sociale des âges de la vie et sur les négociations autour de la détermination des temps de l'existence. Il s'agit de comprendre en quoi les évolutions sociales viennent modifier l'organisation des rythmes de l'existence ${ }^{2}$. 


\section{Cycle de vie et RIG}

4 de rapports de domination entre les âges liés le plus souvent aux transmissions patrimoniales. C'était la règle dans le monde rural et dans le monde de l'aristocratie ${ }^{3}$. dépendaient donc des structures familiales qui étaient elles-mêmes extrêmement diverses d'un bout à l'autre de la France (familles étendues à plusieurs couples recueillant un ou des parents dans le sud, familles souches sous l'autorité du père qui possède l'exploitation dans l'est de la France, famille nucléaire séparant les générations en Île-deFrance et dans l'Ouest).

Le rythme aléatoire des décès donnait la mesure des cycles de l'existence : au début du XVIII siècle, à 15 ans un homme disposait d'une espérance de vie d'environ trente ans contre environ cinquante-sept ans aujourd'hui (soixante-trois ans pour les femmes) ; au $\mathrm{XVIII}^{\mathrm{e}}$ siècle la durée moyenne des unions est inférieure à quinze années : la vie familiale n'avait pas de contenant fixe et n'existait pas sous sa forme présente : les familles étaient en perpétuel éclatement.

7 Les rapports entre individus d'âges différents étaient donc extrêmement variables du fait de l'imprévisibilité des événements démographiques et en particulier des aléas des décès.

Comme le montre Hervé Le Bras ${ }^{4}$ l'image de la famille "tour à plusieurs étages » où aucune génération ne recouvre la suivante est une construction récente qui s'oppose à celle de l'ancien régime démographique où l'on pouvait avoir le même âge que ses oncles ou neveux et où donc les différences de générations ne se repéraient pas par l'âge ${ }^{5}$.

L'allongement de l'espérance de. vie et la baisse de la mortalité (progressive depuis le début $d u \mathrm{XVIII}^{\mathrm{e}}$ siècle et rapide depuis 1945), un meilleur contrôle des calendriers des naissances, la structuration de la famille sur un modèle «bourgeois » à partir du milieu du XIx $x^{e}$ siècle et l'intervention grandissante de l'État dans une politique des âges entraîne dans le même temps une réorganisation des rapports entre les générations et une plus forte structuration institutionnelle et symbolique des cycles de vie.

De ce fait, l'âge est devenu une des composantes essentielles de l'identité. Avec le sexe, l'appartenance à un milieu social et la localisation, c'est le plus puissant point de repère individuel. Il n'est qu'à penser au nombre considérable d'ouvrages ou de revues qui s'adressent (qui ciblent) des catégories d'âges, et où l'on apprend comment être femme à 40 ou à 50 ans ou encore à passer la « middle-life crisis " pour les hommes...

Dans ces conditions on voit apparaître toutes sortes de naturalisation des âges de la vie.

cept de cycle de vie se construit alors sur l'image d'une succession de phases que les individus parcourent automatiquement suivant les étapes d'une socialisation conduisant à la maturité, puis du vieillissement conduisant à la mort. Le déroulement individuel de l'existence est, de fait, scandé par de tels processus, par des passages et des ruptures. Mais le risque est de ne plus penser le déroulement de l'existence que suivant les principes d'une sociologie génétique focalisée sur les aventures de la socialisation individuelle ${ }^{6}$.

13 Les travaux de Daniel J. Levinson sont l'expression la plus achevée d'une telle tendance ${ }^{7}$. Dans la lignée des travaux de Carl C. Jung, Erik H. Erikson, José Ortega y Gasset, l'auteur se 
propose à partir de quarante entretiens de définir le modèle sous-jacent des étapes «standard » qui organisent le déroulement de l'existence. Le modèle est constitué d'une alternance de segments stables et de périodes de transitions advenant à des âges constants (à quatre ou cinq années près).

Une période stable commence lorsque se redéfinit un "pattern"stable de nouvelles tâches à entreprendre. Les événements importants, plus ou moins aléatoires, qui peuvent advenir au cours du déroulement de l'existence ne sont pas de nature à modifier le modèle. Ils prennent leur sens, au contraire, à partir de la place qu'ils y occupent.

Il n'est pas dans notre propos de développer ici la critique interne d'un tel schéma ${ }^{8}$. On insistera plutôt sur le fait, d'une part, que cette analyse ne fait à aucun moment intervenir la question des variations historiques de l'organisation des cycles de vie dans une société donnée et donc dans l'histoire de chaque génération, et, d'autre part, que la définition des âges de la vie dépend de la manière dont une société crée ses concepts d'âge en distribuant les rôles entre les différentes catégories d'âge. Nous pensons pour notre part que les âges sont à considérer dans leur ensemble et qu'il sont à définir les uns par rapport aux autres.

16 Les étapes de l'existence sont scandées par des rythmes historiques extérieurs à ceux du développement des individus comme par exemple la définition sociale des seuils d'âges. Ces définitions ne découlent pas de la simple imposition d'un ordre culturel et sont régies par les places et fonctions assignées aux différents âges de la vie.

Il faut alors chercher quelles nouvelles donnes intergénérationnelles se mettent en place sans céder aux simplifications et au pathétisme de la thèse de la guerre idéologique entre générations qui réifie de nouveaux sujets de l'histoire, ni inversement, à la thèse selon laquelle la réconciliation entre les générations passerait par une bonne pédagogie et une forte motivation à engager la relation d'écoute entre jeunes et adultes.

Pour cela il faut éclaircir ce concept de rapports sociaux intergénérationnels (RIG). Les RIG n'existent jamais à l'état pur. Il s'agit d'une fiction théorique nécessaire. Ils sont toujours articulés, comme nous allons le voir, à d'autres types de rapports sociaux, comme les rapports sociaux de parenté, de classe, de sexe... et ils s'inscrivent dans des systèmes de représentations imaginaires. Ceci dit, nous pensons qu'il n'est pas inutile d'user de ce concept pour rendre compte d'un certain nombre de régulations sociales propres à l'organisation des cycles de vie.

19 En parlant de rapports sociaux intergénérationnels on évitera la traditionnelle opposition entre jeunes et adultes ou entre jeunes et vieux. Parler d'adultes en général consiste à amalgamer des individus situés à des moments fort divers de leur cycle de vie et à renforcer artificiellement l'idée d'une opposition entre classes d'âges (les jeunes contre les adultes) ${ }^{9}$.

20 La définition de rapports intergénérationneis ne suppose pas de découpage a priori des âges de la vie. Elle ne peut en aucun cas être réduite à son acception démographique qui implique la définition d'un écart fixe de vingt-cinq ans pour définir deux générations l'une par rapport à l'autre. Bref, dans cette perspective, on ne part pas d'une définition préalable des âges de la vie pour définir ensuite les rapports entre ces groupes d'âge, mais on part de la définition de rapports sociaux qui mettent en présence des groupes situés à des moments distincts de leur cycle de vie pour comprendre la dynamique de constitution des âges. 
21 La dimension structurelle de ce concept est évidente. On peut le considérer de divers point de vue. L'un d'entre eux est que la reproduction d'une société et la reproduction des classes sociales et les processus de mobilité sociale qui leur sont liés passent par des RIG. Un autre point de vue est que chaque génération est tour à tour débitrice et créancière vis-à-vis des autres et la régulation de ces flux de revenu social est bien au bout du compte une régulation des rapports économiques entre catégories d'âge. Enfin, les rapports intergénérationneis peuvent être abordés du point de vue des interactions pratiques entre agents sociaux. On peut penser aux rapports entre parents et enfants; aux rapports pédagogiques propres à l'école dans sa double dimension d'apprentissage et de soumission à la sélection sociale; aux rapports d'apprentissage ou aux rapports hiérarchiques entre générations sur le lieu de travail... Dans chaque cas il faut déterminer les enjeux, et les formes de domination qu'ils recèlent. Plutôt que de donner une définition théorique générale de ces rapports sociaux nous insisterons, tout d'abord, sur une de leurs caractéristiques théoriques majeures, puis nous utiliserons ce concept de manière heuristique en montrant certaines de ses dimensions.

\section{Occupation des places et socialisation : deux faces des rapports intergénérationnels}

Les RIG se définissent par une sorte de dualité. Ils sont, d'une part, des rapports de socialisation et de transmission et, d'autre part, des rapports qui mettent en opposition des groupes sociaux à propos de la distribution de l'occupation des places sociales entre les différentes catégories d'âge.

On l'a compris, l'intérêt serait de mener l'analyse des situations concrètes à partir de ces deux dimensions à la fois. À ce stade de notre travail nous conservons encore la distinction pour essayer de clarifier le problème.

Le plus souvent on ne retient qu'un aspect des RIG : celui par lequel les jeunes reçoivent des plus anciens. C'est le cas de la plupart des études sur la famille où selon le modèle «éducationnel» le plus simpliste, les enfants sont toujours considérés comme réceptionnaires de savoirs, de biens matériels, d'éducation et rarement comme partie prenante d'un rapport social.

Or, les rapports intergénérationnels sont ambivalents sur le plan individuel et contradictoires sur un plan plus structurel.

Ils s'organisent, d'une part, autour de l'accompagnement et de la protection des plus jeunes (une société qui n'assume plus cette fonction ne peut plus se survivre) et, d'autre part, autour des conflits qui naissent à propos de l'occupation des places sociales. Il faut à la fois que les jeunes épousent les modèles d'identité forgés par les générations antérieures et qu'ils attendent avant d'occuper les places.

29 D'une part, les RIG se définissent comme des rapports de solidarité et, d'autre part, ils se définissent comme des rapports de concurrence à propos de la distribution générationnelle des places sociales.

Concernant les conflits pour l'occupation des places, on a à l'esprit les développements théoriques de Pierre Bourdieu. Les oppositions entre les générations confronteraient dans un champ social donné les détenteurs de biens ou de position sociales (richesses économiques, pouvoir, capitaux symboliques) aux prétendants à ces mêmes biens ou 
places. Suivant les champs, les conflits entre générations seraient d'autant plus grands que des changements importants seraient intervenus dans la définition des postes et des manières d'y accéder.

31 On mesure l'intérêt de cette démarche. Elle fournit une définition générale du rapport entre les générations. Elle permet d'éviter toute définition empiriste des termes du rapport. Elle peut s'appliquer à toute sorte de situations ou de champs sociaux et de repérer l'effet propre des rapports intergénérationnels. Elle permet de penser les rapports entre dynamique des rapports intergénérationnels et organisation du cycle de vie. Ainsi, la détermination des moments et des modalités des passages entre les différents moments de l'existence peut être l'objet de conflits entre détenteurs et prétendants. Et ce, même dans les sociétés où la très forte ritualisation des passages pourrait laisser croire que le choix de leur moment est invariable d'une génération à l'autre et que leur haute teneur symbolique résout les conflits proprement politiques relatifs à l'occupation des places.

L'importance de l'âge dans la détermination de la position sociale et de nouvelles phases du cycle de vie varie suivant les types de sociétés. Il existe des sociétés où pouvoir, richesse et accès aux ressources sont très fortement liés à l'appartenance à un groupe d'âge ( age-set societies»). Le plus généralement, le pouvoir appartient aux plus âgés jusqu'à une certaine limite d'âge (principe de la gérontocratie). Dans ces sociétés les critères de passage d'un groupe d'âge à un autre sont particulièrement cruciaux ${ }^{10}$.

La question des passages entre les différents moments du cycle de vie constitue un des points de débat sur la socialisation et les modalités de déroulement du cours de l'existence.

Selon les cas, on met plutôt l'accent sur :

- la fonction de régulation macro-sociale, où le passage est conçu comme un acte de légitimation sociale d'une nouvelle position sociale ;

- la fonction de régulation individuelle des rites de passage et d'accompagnement du processus de maturation;

- le sens des rythmes ainsi imposés aux individus ;

- les diverses configurations intergénérationnelles de l'existence selon la position sur le cycle de vie.

Dans la première perspective Pierre Bourdieu insiste plus sur la ligne de démarcation que constitue le rite que sur le passage lui même. Le rite de passage est, selon lui, l'expression de cette magie sociale qui consiste à produire du discontinu avec du continu (on a à l'esprit l'exemple du concours et de la ligne qu'il institue entre le dernier reçu et le premier recalé). Le rite dans cette perspective est un acte d'institution ou un acte d'investiture qui assigne une essence sociale, un destin pour l'éternité. Que l'on pense aux diplômés des grandes écoles qui une fois pour toutes occuperont les places «qui leur reviennent ».

La seconde perspective amène à se centrer sur les modalités même du rituel pour y lire les modalités de l'accompagnement psychologique de l'individu. On peut dans cette perspective analyser les variations des formes et de la nature des passages suivant les classes, les sexes, les régions, les ethnies. Van Gennep a pour sa part postulé que ces variations se jouaient autour d'une forme récurrente où l'on retrouvait les éléments suivants: une période d'isolement (séparation/retraite) - une épreuve - une mort symbolique - un passeur - un langage initiatique et une référence à un ancêtre mythique 
- une renaissance symbolique, sorte de deuxième naissance - un retour à la communauté sous un autre statut.

De là on mettra en avant des invariants anthropologiques ou en tout cas des matrices symboliques de très longue durée : «Tous les rituels mettent en scène un passage par la mort qui apparait comme la condition essentielle de l'accès au pouvoir ${ }^{11}$. On voit le débat s'ouvrir ici largement avec d'autres thèses comme celles de $P$. Ariès qui pour sa part rejette absolument la thèse selon laquelle les rites d'initiation de l'adolescence seraient une constante de toutes les sociétés humaines et selon laquelle ils suivraient une même structure rituelle.

Ceux qui mettent en avant la gestion des rapports de pouvoir intergénérationnels dans une société sont amenés à mettre l'accent sur le fait que chaque nouvelle génération se trouve confrontée à de nouveaux problèmes selon les équilibres économiques, démographiques, politiques du moment et donc que les passages varient suivant les problèmes que chaque génération doit résoudre.

De ce point de vue, il faudrait moduler les oppositions tranchées entre les sociétés « traditionnelles » qui accompagneraient les impétrants par des rites qui manifesteraient le soutien de la communauté et les nôtres qui abandonneraient leurs enfants sous prétexte qu'elles n'accompagneraient plus les passages par des rites.

Dans les sociétés basées sur les groupes d'âge les rites de passage sont faits à la fois de la brutalité de l'imposition d'un ordre social et de l'accompagnement des jeunes par leur entourage. Mais les adultes font en sorte de rendre compatibles les exigences d'ordre social et celles qui sont liées à la maturation... Mais alors que des anthropologues comme R. Benedict n'avaient vu que le rôle de soutien des groupes de pairs, d'autres anthropologues ${ }^{12}$ ont su comprendre ce double processus d'accompagnement et de conflit : les conflits ne sont pas rares dans les age-set societies à propos de la définition du moment des passages. Ce moment peut varier suivant l'équilibre des forces politiques dans ces sociétés.

41 Les rites se perdent et l'on assiste à un mouvement de déconnexion des moments d'apparition des attributs de la maturité : les uns comme la maturité sexuelle se situent à des âges plus précoces, d'autres comme la maturité professionnelle et l'indépendance financière à des âges plus tardifs.

En fait, il ne faut pas mythifier les sociétés traditionnelles. La ritualisation des passages enfermait les individus dans des rôles sociaux dont ils ne sortaient que très difficilement. L'intérêt des passages plus longs est aussi de ne pas cristalliser trop tôt l'assignation à des places et de laisser les possibles ouverts plus longtemps. Quand passé de l'enfant et avenir de l'adulte se superposent sur le présent de l'adolescent, il ne reste rien au présent pour les jeux d'anticipation imaginaire et d'émancipation du poids d'Anchise.

\section{De quelques lieux décisifs où lire des rapports intergénérationnels}

\subsection{Rapports intergénérationnels et rapports de classes}

43 L'analyse des rapports intergénérationnels peut permettre de repenser les processus de reproduction de chaque classe sociale, en particulier en réintroduisant la dimension contradictoire et conflictuelle de ces processus contrairement à l'imagerie répandue de la 
transmission du relais au cours des générations. Car si le relais ne se transmet pas au bon moment, si par exemple il se transmet trop tard, c'est-à-dire à un moment où la génération qui suit est à une étape de son cycle de vie où elle a déjà « fait sa vie », alors il n'y a plus personne à qui transmettre le relais.

On a en mémoire les conflits qui dans le monde paysan ont opposé les pères aux fils. Les fils refusant de plus en plus le pouvoir discrétionnaire des pères et le statut d'aide familial qui leur était imposé jusqu'à la prise de retraite des pères. Ce conflit a même pris une forme collective avec la création du CNJA en 1956 et la publication de l'ouvrage de Michel Debatisse $^{13}$. Les jeunes générations de paysans ne veulent plus attendre aussi longtemps les passations de pouvoir et demandent leur reconnaissance au statut d'associé au chef d'exploitation (lois de 1973 et 1974). Bien des éléments peuvent expliquer le renversement du rapport de force et en particulier la mise en place du régime de retraite des paysans (1952). Il est à cet égard intéressant de constater que dans ce cas c'est une transformation étatique dans l'organisation des cycles de vie qui va contribuer à transformer les termes du rapport de force intergénérationnel dans la famille.

Cette analyse peut être étendue à d'autres classes ou catégories sociales où la reproduction de la classe et les rapports professionnels intergénérationnels ne passent pas par des transmissions de patrimoine. Ainsi, sur le marché de l'emploi externe, on a pu constater que les mesures adoptées en faveur de l'emploi des jeunes par le gouvernement de Jacques Chirac (exonération de charges pour les entreprises,...) ont eu pour effet d'accélérer les licenciements dans les autres classes d'âge. Ce qui s'appelle déshabiller les pères pour (mal) habiller les fils.

Les politiques salariales organisent donc la concurrence entre les âges. Mais il faudrait en fait ici pouvoir distinguer finement les catégories socio-professionnelles par âge (ex : les préretraités, les nouveaux embauchés, les inactifs jeunes, etc.).

47 On sait déjà que les employeurs évaluent la qualification autant par le diplôme que par le cheminement professionnel et personnel en définissant par là même les termes de la concurrence entre les primo-demandeurs, les jeunes chômeurs et les travailleurs expérimentés à la recherche d'une mobilité. On sait que souvent ils considèrent autant la qualification que la situation sur le cycle de vie : nous pensons à la concurrence entre les jeunes filles avec les femmes qui reprennent un emploi après les naissances.

48 Si l'on rappelle par ailleurs que la mobilité professionnelle dans les milieux ouvriers a notoirement baissé depuis dix ans, on mesure l'âpreté nouvelle de la lutte pour l'occupation des places professionnelles. Le déroulement de la vie professionnelle en est alors principalement affecté par le fait que certains groupes d'âge font l'objet de régulations salariales spécifiques: les jeunes, avec tous les systèmes de salaires situés sous le niveau du SMIC comme les TUC, et les plus vieux avec l'introduction de systèmes de préretraites ou de sous-SMIC, à l'instar des plus jeunes, avec le système des PIL ou avec l'introduction de «passage à la retraite en biseau » et la diffusion des temps partiels pour futurs retraités.

On n'oubliera pas ici que la modification des cycles de vie professionnels est marquée par l'histoire démographique des générations. Le "dégraissage» de la quarantaine correspond aussi au vieillissement des embauches massives des jeunes entre 1965 et 1973 14 . 

que la situation des pères qui barraient la route aux fils n'a guère plus cours ${ }^{16}$. La solidarité tend à remplacer la concurrence qui se déplace ailleurs. Mais on sait finalement peu de chose sur les négociations internes à la famille et sur les phénomènes de solidarité à double sens des parents vers les enfants et des enfants vers les parents. Les enjeux de ces rapports varient avec les générations et de nouvelles zones de négociations ou de conflits peuvent apparaître. Ainsi, dans une période de crise, la famille est de plus en plus sollicitée. De ce point de vue les tendances en cours peuvent déboucher autant sur le renforcement des solidarités que sur leur éclatement. 
55 À propos par exemple de la détermination du moment où les jeunes doivent quitter le foyer parental ${ }^{17}$. Ainsi, jusqu'à une période somme toute encore peu éloignée, les parents se mêlaient de très près des conditions dans lesquelles les jeunes prenaient leur autonomie. Ils le faisaient de plusieurs manières suivant les classes et les sexes.

Certains historiens nous expliquent comment pour les garçons, dans le monde rural en particulier, certaines règles implicites réglaient les relations économiques et les conditions du départ des garçons. Au retour du service militaire, le garçon pouvait rester encore un ou deux ans sur l'exploitation familiale en échange de l'aide de la famille pendant la conscription ${ }^{18}$

D'autres montrent comment pour les familles populaires l'arrivée de la paye des enfants améliorait les conditions de vie des familles. La mobilisation de nouvelles ressources de la famille permettait pour cette dernière de connaître une phase relativement plus aisée de son histoire ${ }^{19}$.

On pense également à la prise d'autonomie des jeunes bourgeoises: le futur conjoint devait avoir " une situation », ou tout au moins un métier. Le père ne laissait pas sa fille partir à n'importe quelle condition avec n'importe qui : on a présent à l'esprit les drames familiaux qui furent un des thèmes privilégiés de la littérature ayant la famille pour objet. Jusqu'où, selon les milieux, cette situation perdure-t-elle encore et sous quelles formes? Dans les cas où le consensus familial est encore nécessaire, quelles formes revêt-il ? Dans quelle mesure la décision concernant le moment de la décohabitation est-elle encore l'affaire de la famille? Les conflits n'apparaissent-ils pas précisément lorsque les critères auxquels se réfèrent les jeunes gens et les jeunes filles sont différents de ceux de leurs parents?

La caractéristique des échanges familiaux est qu'ils agissent sur la durée de l'existence. La logique de l'échange familial peut ainsi s'étaler sur l'ensemble du cycle de vie. Est-il besoin de rappeler que le principe du contre-don est de travailler dans le temps?

Lorsqu'on analyse la filiation, on insiste sur les formes d'investissements économiques, culturels, sociaux, dont les enfants font l'objet, ou encore sur les formes de socialisation, les transmissions de valeurs ou de patrimoine, sur les stratégies de fécondité ou encore sur les différenciations que les parents opèrent au sein de la fratrie, mais on ne sait que très peu de choses sur les formes de négociation qui peuvent apparaitre, et donc sur la capacité d'intervention active des jeunes dans cette négociation.

61 Nous avons montré par ailleurs ${ }^{20}$ que les fils actifs ayant un emploi, dont le père était chômeur, restaient beaucoup plus longtemps au domicile familial que les fils actifs ayant un emploi, dont le père avait lui-même un emploi. De nouvelles formes de solidarités familiales voient peut-être le jour. Non plus celles qui prenaient la forme de la paie rapportée sur la table, c'est-à-dire celles basées sur une autorité familiale s'imposant à un jeune pour lequel cohabitation était synonyme de dépendance, mais solidarités entre hommes de la famille.

Il faut donc aussi comprendre à quel moment de leur cycle d'existence, et donc de leur cursus résidentiel, se trouvent eux-mêmes les parents (ils ont généralement entre 40 et 50 ans lorsque le problème du départ des enfants se pose). Quelles sont alors les conditions requises pour engager un processus de mobilisation pour aider les enfants à entrer dans la vie? Que ou qui sacrifie-t-on d'autre? La capacité à se mobiliser dans le présent passe bien souvent par une sorte de consensus familial sur la représentation de l'avenir, une sorte de conscience de lignée en quelque sorte: comment se transforme-t- 
elle, lorsqu'elle existe, en ce moment crucial de l'existence des parents, comme des enfants, où chacun peut faire les comptes d'une cohabitation déjà longue?

On vient de souligner les solidarités, mais il existe aussi des tensions intergénérationnelles. Elles apparaissent dans une situation où la décohabitation plus tardive et le décalage entre les différents attributs de la maturité sont exacerbés. Quelles relations nouvelles peuvent s'établir entre des parents et leurs enfants, déjà adultes, encore enfants, bref, dont le statut d'âge et de maturité socialement reconnu est très incertain? Ici les rapports intergénérationnels changent de sens sous le double effet de l'évolution des rapports de parenté et des éthiques qui leurs sont liées, et des nouvelles conditions économiques qui modifient les modes d'entrée dans la vie active et définissent de nouvelles fonctions à la famille.

La redistribution des rôles générationnels dépend fortement de l'histoire de chaque génération. Là comme ailleurs on ne peut raisonner sur des générations abstraites. On prendra deux exemples.

Le premier concerne le monde ouvrier à la fin du XIX ${ }^{\mathrm{e}}$ siècle. Ronald Melchers ${ }^{21}$ montre à ce propos que dans une entreprise telle que Schneider au Creusot, où les ouvriers travaillent de père en fils pour le même employeur, celui-ci est amené à opérer un certain traitement social de la vieillesse ouvrière avec le maintien des travailleurs âgés dans des emplois adaptés à leurs forces et l'instauration d'un système de retraite, sous la pression non pas des ouvriers âgés mais de leurs enfants qui se montreraient plus exigeants en matière de salaires s'ils devaient avoir leurs parents à charge. On le voit, les rapports entre systèmes institutionnalisés de retraite et solidarités familiales sont très étroits. On ne peut de ce fait autonomiser la sphère des rapports familiaux intergénérationnels.

Le second se formulera sous forme d'hypothèse et concerne les générations de l'aprèsguerre. Si on considère la (ou les) génération née dans l'entre-deux-guerres on constate que cette (ou ces) génération a vu ses habitudes de consommation se forger dans le cadre d'une économie relativement stagnante et à partir d'habitudes d'auto-production familiales : on reprisait les chaussettes, on récupérait les bouts de ficelles, on mangeait beaucoup de pain, dans la majorité des ménages. La guerre renforcera encore ces comportements.

67 Cette (ou ces) génération donne naissance à la génération qui naît entre, grosso modo, 1945 et la fin des années 1950. À mesure que ses enfants grandissaient au long des années de forte croissance, de 1950 au début des années 1970, cette génération a vu son pouvoir d'achat s'accroître. L'allocation de ces ressources nouvelles a fait l'objet d'arbitrages nouveaux de leur part: on pouvait consommer davantage pour soi (voiture, vêtements, vacances...), on pouvait aussi consacrer davantage à la génération suivante, en particulier en finançant une plus longue scolarité aux enfants. Concrètement, on a pu faire les deux ; mais nous faisons l'hypothèse que l'hystérésis des habitus de consommation acquis avant 1950 a favorisé un certain altruisme intergénérationnel. La mobilisation des parents en faveur de leurs enfants (études, aide à l'établissement des jeunes ménages...) était d'autant plus facile que la conjonction de l'accroissement des revenus et la subsistance des pratiques domestiques antérieures à cet accroissement dégageait une épargne. Les investissements scolaires étaient payés de retour, dans une période de développement de l'appareil scolaire et de relative adéquation des filières professionnelles aux diplômes.

68 Cette génération du baby-boom fera-t-elle preuve d'un même altruisme vis-à-vis de ses propres enfants? En tout cas, elle en aura moins. Mais une fois passée aux statuts de 
papies et de mamies, assurera-t-elle les mêmes fonctions que les grands-parents de la génération précédente? Peut-être pas! Et ce, du fait à la fois qu'elle n'a pas connu la même histoire consommatoire, que les femmes ont connu plus massivement d'autres statuts sociaux que celui de mère et que le statut de ce nouvel âge de la vie a changé pour être plus ouvert à des activités extra-familiales ${ }^{22}$.$$
\text { large de la redistribution du revenu social où chaque génération est tour à tour débitrice }
$$
et créancière ${ }^{24}$.

77 Le développement du welfare-state a amplifié le système de. quadrillage des âges en particulier pour mieux définir les règles de redistribution du revenu social entre 
générations. La définition de la règle des 37,5 ans pour avoir accès à une retraite complète renvoie à une telle opération de redistribution. De plus, dès sa naissance, l'individu est pris dans un réseau de règles juridiques basées sur des critères d'âge: depuis l'âge de 3 ans le parcours scolaire est très strictement encadré par des limites d'âge ; on relève de la pédiatrie jusqu'à 15 ans; il faut avoir 13 ans pour voir certains films, 16 ans pour avoir le droit de conduire une moto ; les programmes de formation sont réservés à certaines catégories d'âge (16-18 ans, 18-21 ans...). Plus tard les ouvertures de droits sont également soumis à des critères d'âge : les " cartes jeunes ", les « cartes vermeil », les réductions en avion, l'autorisation de figurer sur la Sécurité Sociale des parents, les âges limites (supérieurs ou inférieurs) pour être admis sur certains emplois, etc.

Déjà se dessine un nouveau débat qui pose sur une grande échelle la question de la redéfinition des solidarités intergénérationnelles à partir de la question politique des transferts sociaux entre inactifs jeunes, inactifs retraités et actifs. Ne va-t-on pas voir se constituer des groupes de pression fiscaux délimités en fonction de l'âge et de la génération?

Certains auteurs comme Xavier Gaullier ${ }^{25}$ pensent que le moment est venu de poser les bases d'un nouveau contrat social entre générations, moins à partir d'un recentrage des systèmes de répartition sur l'apport contributif que sur une nouvelle répartition sur le cycle de vie des périodes d'activité et d'inactivité professionnelle, avec la mise en place d'un principe de droit de tirage en temps non salarié pour la formation et les loisirs selon un ratio de points de cotisations acquises.

On le voit, la pyramide des âges n'est pas en sol un problème. Le problème est avant tout celui de la redéfinition concommitante du rapport salarial et du cycle de vie.

On aperçoit aussi le lien entre politique sociale et rapports intergénérationnels dans la famille. On ne peut, en effet, aussi simplement garder la solidarité pour les relations familiales et déplacer le conflit intergénérationnel sur les politiques sociales. En tout cas, il ne faut pas clore le débat ${ }^{26}$.

La sensiblerie intergénérationnelle peut aussi cacher un projet politique. Celui qui consiste à réserver les financements publics pour le soutien aux firmes dans une situation de concurrence internationale (la thèse du bouclier industriel) et à transférer une partie des fonctions sociales remplies dans le cadre du welfare state. Les solidarités intergénérationnelles ont alors bon dos : déjà on y voit le modèle des nouvelles solidarités de proximité et l'amorce de nouvelle formes de production sociale non marchande. Pourquoi pas? Mais la sociologie est toujours perdante à vouloir arrondir les bords des rapports sociaux et se délester de sa fonction de lucidité.

\section{Conclusion}

L'évocation de ces quelques problèmes nous montre qu'il n'est pas possible d'analyser chaque âge de la vie comme un univers social spécifique ou un groupe homogène. L'utilisation métaphorique de la notion de génération (la génération de 1968, la bof génération, etc.) ne doit pas faire oublier que ce sont les rapports qui définissent les termes du rapport et les équilibres généraux des rapports entre les âges qui définissent les enjeux des rapports entre les générations.

De même, il apparait que le cours de la vie dépend des rapports proprement historiques qui lient chaque génération. Dernier exemple : dans l'entre-deux-guerres les pertes de la 
"génération du feu » ont laissé des places vacantes auxquelles les jeunes ont pu accéder. Mais plus encore, l'ensemble du corps social inculque le respect d'aînés qui ont gagné la guerre, qui ont souffert, qui ont donné un million et demi de morts. On ne se révoltait pas contre une telle génération ${ }^{27}$. Ces générations chantaient encore la Marseillaise : « Nous entrerons dans la carrière ${ }^{28}$ quand nos aînés n'y seront plus... ». Elles ont oublié ce couplet.

Enfin, l'évolution de la définition des âges de la vie résulte également des rapports de force idéologiques qui à chaque époque façonnent la conception globale du cycle de vie. On pourrait de ce point de vue, et pour conclure de manière prospective, distinguer trois modèles idéologiques correspondant à trois conceptions des temporalités de l'existence.

Les premières insistent sur les exigences propres du processus de socialisation avec la reconnaissance d'un droit a prendre le temps qu'il faut pour se socialiser. On peut ici insister soit sur l'allongement des temporalités de passage à l'état adulte comme une avancée en ce sens (si on ne réduit pas ce processus au seul effet de la crise économique); soit, inversement, on insiste sur le fait que la logique scolaire est de plus en plus exigente sur le plan temporel, le resserrement considérable de la dispersion des âges des élèves de seconde des lycées étant un exemple très symptomatique de la pression sociale sur le rythme propre des processus de socialisation individuelle ${ }^{29}$.

On peut ici remettre en cause les nouvelles formes de concurrence intragénérationnelle et l'imposition de seuils d'âge qui leur sont liés au nom des impératifs de la socialisation individuelle. On peut aussi élargir la perspective et poser le problème plus général d'une nouvelle flexibilité temporelle dans l'organisation de l'existence. La redistribution des temps de formation au cours de l'existence vient d'ailleurs maintenant se constituer en problème politique majeur.

On distinguera ensuite les conceptions inspirées du juridisme et du légalisme temporel du welfare state qui s'en tiennent au constat de l'existence d'un temps pour chaque chose et de temporalités fixées par des règles institutionnelles. Ici, le temps est assigné par le jeu d'échéances limites. La dimension politique de l'affectation sociale des âges de la vie et les arbitrages qui ont conduit à les définir, par la fixation de seuils par exemple, sont occultés et les contraintes fortes liées à l'âge ne sont pas remises en cause: le temps institutionnalisé est proprement fétichisé. Il devient une seconde nature sociale.

Enfin, il y a les conceptions qui, au nom des valeurs «libérales » de l'accomplissement personnel envers et contre tous, instituent la précocité comme valeur suprême de la distinction au détriment des rythmes de socialisation propre à l'histoire de chacun.

Ces valeurs d'achievement conduisent en fait à considérer le cycle de vie comme une courbe de carrière ascendante sur le modèle du cadre d'entreprise. On voit alors le danger de voir naitre des formes de concurrence exacerbées entre les générations. La boutade familière du "place aux jeunes" risque alors de devenir une méchante machine idéologique justifiant le "dégraissage " des effectifs des personnes les plus âgées. L'idéologie de la concurrence généralisée s'applique au RIG.

91 Un ouvrage récent dénonce avec virulence le rôle rédempteur que les «sociétés postmodernes » veulent faire jouer à la jeunesse ${ }^{30}$. Il faut admettre qu'est posé là un sérieux problème.

Dans nos sociétés on peut penser que la question de la détermination des seuils d'âge prend et prendra une importance grandissante. Mais sur quel mode ? Le mode étatique de la définition institutionnelles des seuils d'âge, le mode « libéral » du jeu de l'élitisme par la précocité ou le mode contractuel de la définition des rythmes de l'existence à partir 
d'une définition globale de droits et devoir des diverses générations les unes par rapport aux autres.

On touche ici du doigt le fait que parler de rapports entre générations, c'est faire plus que de parler des rapports entre groupes situés sur des positions différentes du cycle de vie. C'est prendre en considération le fait que ces générations sont porteuses d'histoires, d'éthiques et de représentations du monde différentes. C'est prendre en considération, d'une part la dimension politique des affectations sociales des âges de la vie, et d'autre part les conjonctures historiques particulières sous lesquelles se déroulent ces rapports sociaux.

On constate que les rapports d'influence idéologique se déplacent pour réévaluer les places des jeunes et des post-jeunes (les 25-40 ans) et celle des adultes mûrs. Chaque époque valorise à sa manière les différents groupes d'âges. Mais derrière les expressions moralisées du rapport aux âges de la vie, il faut aussi savoir lire les stratégies de développement social.

On le voit, l'analyse biographique doit aussi être réinscrite sur des enjeux sociétaux de vaste ampleur.

\section{NOTES}

1. Comme premier effort de l'auteur en ce sens, voir : "Quelle génération ? Quelle génération?», communication au Colloque national de la Société d'ethnologie française Classe d'âge et société de jeunesse, Le Creusot, mai 1985.

2. Les travaux de J.-C. Lagrée vont également en ce sens.

3. N.-J. Smelser, S. Halpern, «The historical triangulation of family, economy and education. Turning points. Historical and sociological essays in the family », American Journal of Sociology, 84, 1978.

4. H. Le Bras, «L'évolution des liens de famille au cours de l'existence. Une comparaison entre la France actuelle et la France du XVIII ${ }^{\mathrm{e}}$ siècle ", in Les âges de la vie, Actes du $7^{\mathrm{e}}$ Colloque national de démographie (Strasbourg, 5-7 mai 1982), Paris, Presses universitaires de France, 1982 («Travaux et documents de l'INED ", 96).

5. On ne développera pas ici la dimension anthropologique. Un tel développement impliquerait que l'on s'interroge sur les raisons du déplacement du balancier des relations de filiation vers celles d'alliance et sur celles d'un éventuel retour de balancier. Certains travaux récents abordent cette dimension de manière approfondie comme ceux de Claudine Attias-Donfut, Sociologie des générations. L'empreinte du temps, Paris, Presses universitaires de France, 1988 (« Le sociologue »).

6. Comme exemple récent de pédago-médicalisation de la question des âges de la vie, cf. É. Noël, Les âges de l'homme. Émile Noël s'entretient avec..., Paris, Eshel/France-Culture, 1988.

7. «À developmental approach to the pre-adult years means, too, that we can discern an underlying order in the child's movement through them. It is now generally accepted that all lives are governed by common developmental principles in childhood and adolescence and go through a common sequence of developmental periods [...] A developmental approach is needed in the study of adulthood » (D. J. Levinson, The seasons of a man's life, New York, Alfred A. Knopf, 1978). 
8. Comme exemples de critiques « internes » on pourra mentionner le fait qu'on ne distingue pas les étudiants des salariés dans la période 22-28 ans (" entering the adult world») ou le fait de ne parler des adultes qu'en étudiant les seuls hommes...

9. A. Percheron, «Se faire entendre. Morale quotidienne et attitudes politiques des jeunes », in $\mathrm{H}$. Mendras, ed., La sagesse et le désordre, Paris, Gallimard, 1980 («Bibliothèque des sciences humaines »).

10. A. Fonner, D. D. Kertsen, "Transitions over the life course. Lessons from age-set societies ", American Journal of Sociology, 83 (5), 1978.

11. M. Augé, Pouvoir de vie, pouvoir de mort, Paris, Flammarion, 1977.

12. A. Fonner, D. D. Kertsen, «Transitions over the life course... ».

13. M. Debatisse, La révolution silencieuse. Le combat des paysans, Paris, Calman-Lévy, 1963.

14. X. Gaullier, La deuxième carrière. Âges, emplois, retraites, Paris, Seuil, 1988 ("L'épreuve des faits »).

15. Là encore, il ne pas faut mythifier le passé. La rétention des savoir-faire et les secrets qui entouraient les tours de main constituaient une sorte de rente de situation pour les ouvriers. Ils ne les cédaient pas aussi facilement que cela aux plus jeunes.

16. La question des rapports intergénérationnels dans la famille pose également le problème de l'évolution de la figure du père. La notion de père est complexe. Nous ne savons plus bien ce qu'est un père. Nous voyons en revanche que la forme de famille née vers le XII ${ }^{\mathrm{e}}$ siècle s'achève. La figure paternelle construite au Moyen Âge ( $\mathrm{xI}^{\mathrm{e}}$ siècle) s'écroule. Après le pater familias romain qui a droit de vie ou de mort sur le fils, l'institution du mariage assoit la position de force du père : c'est celui que le mariage désigne. On assiste à de nouvelles formes de paternité inédites dans l'histoire. Il faut reconceptualiser la notion de paternité. La Révolution française avait limité certains pouvoirs du père. Mais le Code civil de 1804 instaure le règne du padre padrone, du père chef d'entreprise. À partir de la fin du XIX ${ }^{\mathrm{e}}$ siècle commence la mutation du statut paternel : une loi de 1889 introduit la notion de déchéance paternelle; en 1912 sont créés des tribunaux pour enfants et l'on commence à développer des enquêtes sociales: on commence à distinguer les «bons » et les « mauvais » pères; 1935 voit l'abolition du droit de correction paternelle ; en 1970 la notion d'autorité parentale remplace celle d'autorité paternelle: la mère peut exercer l'autorité parentale; en 1972 on reconnaît la filiation naturelle : la mère peut déclarer l'enfant sous son nom de jeune fille; plus récemment, on légalise la paternité secrète avec le développement de la technique de l'insémination artificielle avec donneur. Ces changements juridiques révèlent l'apparition d'une nouvelle définition de la paternité et de la filiation. Ils révèlent également une remise en cause de l'image de la paternité toute-puissante. L'apparition de la notion de père carent ou démissionnaire en est le signe le plus net.

Dans chaque société se retrouvent au moins trois modalités de l'exercice de la paternité : celle de géniteur ; celle d'éducateur et de nourricier ; celle d'autorité au nom de laquelle on transmet les noms et les biens. Or, on assiste à la rupture de l'unité des fonctions rassemblées sous un même personnage: géniteur-éducateur-nourricier-donneur de nom-mari de la mère. Dans d'autres sociétés ces fonctions sont réparties sur plusieurs personnes. La nôtre découvre l'éclatement de ces fonctions et leur appropriation par diverses personnes ou institutions.

Sur les évolutions de la famille on peut se reporter à l'ouvrage de G. Duby, Le chevalier, la femme, le prêtre, Paris, Hachette, 1981 ; et F. Hurstel, « Des lois à la Loi », Colloque Fonction paternelle, mai 1987 ; sur les questions de la paternité on pourra consulter F. Delaisi de Parceval, F. Hurstel, « La paternité à la française ", Les Temps modernes, sept. 1986.

17. F. Godard, T. Bloss, « La décohabitation des jeunes », Cahiers de l'INED, 1988.

18. A. Prost, «Mariage, jeunesse et société à Orléans en 1911 », Annales. Économies, Sociétés, Civilisations, 36 (4), 1981, p. 672-701.

19. B. Seebohm Rowntree, Poverty. À study of town life, Londres-New York, Macmillan, 1901.

20. F. Godard, T. Bloss, « La décohabitation des jeunes ». 
21. R.-F. Melchers, La vieillesse ouvrière, 1836-1914. Les origines de la retraite ouvrière, Marseille, Université Aix-Marseille-II, 1984, thèse de $3^{\mathrm{e}}$ cycle.

22. On est loin des certitudes familialistes d'un ouvrage récent qui perçoit un effacement de la relation financière derrière le geste d'amour dans les relations familiales intergénérationnelles : S. Royal, Le printemps des grands-parents. La nouvelle alliance des âges, Paris, Cogite/Robert Laffont, 1987.

23. M. Halbwachs, Classes sociales et morphologie, Paris, Minuit, 1972 (« Le sens commun »).

24. J.-C. Chesnay, «Les inégalités économiques entre générations ", in D. Kessler \& A. masson, eds, Cycles de vie et générations, Paris, Economica, 1985.

25. X. Gaullier, La deuxième carrière..., p. 174-182. Sur ce point, on peut aussi consulter l'ouvrage d'Alain Lipietz, L'audace ou l'enlisement. Sur les politiques économiques de la gauche, Paris, La Découverte, 1984.

26. Comme le fait Ségolène Royal dans Le printemps des grands-parents.

27. A. Prost, "Youth protest and every day culture in France between the wars », Colloque Jugend Protest und Generation Enkondikt in Deutschland, Frankreich und Italienun, 20. Jahrhundert Brunswick, juin 1985.

28. Il s'agit ici de la carrière où l'on entraîne les chevaux. Rouget de Lisle se réfère ici au rôle de la maîtrise des chevaux dans l'institution de l'éphébie dans la Grèce antique (cf. P. Vidal-Naquet, Le chasseur noir. Problème de l'éphébie en Grèce antique, Paris, Maspero, 1978). L'éphébie a disparu et on ne fait pas de contre-sens en pensant à la carrière professionnelle.

29. A. Prost, L'enseignement s'est-il démocratisé ?, Paris, Presses universitaires de France, 1986.

30. A. Finkielkraut, La défaite de la pensée, Paris, Gallimard, 1987 (« Essai »). 